Article

\title{
Fluorescence and Physico-Chemical Properties of Hydrogenated Detonation Nanodiamonds
}

\author{
Giannis Thalassinos ${ }^{1, *(1)}$, Alastair Stacey ${ }^{1}\left(\mathbb{D}\right.$, Nikolai Dontschuk $^{2}\left(\mathbb{D}\right.$, Billy J. $_{\text {Murdoch }}{ }^{3}(\mathbb{D}$, \\ Edwin Mayes $\left.{ }^{3}{ }^{(}\right)$, Hugues A. Girard $\left.{ }^{4}{ }^{(}\right)$, Ibrahim M. Abdullahi ${ }^{5}$, Lars Thomsen $\left.{ }^{6}{ }^{(}\right)$, \\ Anton Tadich ${ }^{6}{ }^{\circledR}$, Jean-Charles Arnault ${ }^{4}{ }^{\circledR}$, Vadym N. Mochalin ${ }^{5, *}{ }^{\circledR}$ and Brant C. Gibson ${ }^{1}$ \\ and Philipp Reineck ${ }^{1, *}$ (i) \\ 1 ARC Centre of Excellence for Nanoscale BioPhotonics, School of Science, RMIT University, \\ Melbourne, VIC 3001, Australia; alastair.stacey@rmit.edu.au (A.S.); brant.gibson@rmit.edu.au (B.C.G.) \\ 2 Centre for Quantum Computational and Communication Technology, School of Physics, University of \\ Melbourne, Melbourne, VIC 3010, Australia; dontschuk.n@unimelb.edu.au \\ 3 RMIT Microscopy \& Microanalysis Facility, RMIT University, Melbourne, VIC 3001, Australia; \\ billy.murdoch@rmit.edu.au (B.J.M.); edwin.mayes@rmit.edu.au (E.M.) \\ 4 CEA, LIST, Diamond Sensors Laboratory, F-91191 Gif-sur-Yvette, France; hugues.girard@cea.fr (H.A.G.); \\ Jean-Charles.ARNAULT@cea.fr (J.-C.A.) \\ 5 Department of Chemistry and Department of Materials Science \& Engineering, Missouri University of \\ Science and Technology, Rolla, MO 65409, USA; ima284@mst.edu \\ 6 Australian Synchrotron, ANSTO, Clayton, VIC 3168, Australia; larst@ansto.gov.au (L.T.); \\ antont@ansto.gov.au (A.T.) \\ * Correspondence: giannis.thalassinos@rmit.edu.au (G.T.); mochalinv@mst.edu (V.N.M.); \\ philipp.reineck@rmit.edu.au (P.R.)
}

Received: 31 December 2019; Accepted: 31 January 2020 ; Published: 7 February 2020

\begin{abstract}
Hydrogenated detonation nanodiamonds are of great interest for emerging applications in areas from biology and medicine to lubrication. Here, we compare the two main hydrogenation techniques-annealing in hydrogen and plasma-assisted hydrogenation-for the creation of detonation nanodiamonds with a hydrogen terminated surface from the same starting material. Synchrotron-based soft X-ray spectroscopy, infrared absorption spectroscopy, and electron energy loss spectroscopy were employed to quantify diamond and non-diamond carbon contents and determine the surface chemistries of all samples. Dynamic light scattering was used to study the particles' colloidal properties in water. For the first time, steady-state and time-resolved fluorescence spectroscopy analysis at temperatures from room temperature down to $10 \mathrm{~K}$ was performed to investigate the particles' fluorescence properties. Our results show that both hydrogenation techniques produce hydrogenated detonation nanodiamonds with overall similar physico-chemical and fluorescence properties.
\end{abstract}

Keywords: detonation nanodiamond; hydrogenation; graphitization; surface chemistry; fluorescence; TEM; NEXAFS; XPS; FTIR; DLS

\section{Introduction}

Detonation nanodiamonds (DNDs) have attracted great attention in the scientific and industrial communities in the past decade [1,2]. Today, DNDs are already evaluated in applications from composite materials in dental implants [3] and drug delivery applications [4] to diamond growth [5] and lubrication [6]. At the same time, many questions about their exact nanoscale composition, crystal structure, and surface properties remain unanswered, necessitating continued efforts in the detailed material characterization of DNDs. 
The hydrogenation of diamond is of great interest, as it imparts a p-type surface conductivity to bulk diamond samples [7] and also increases the electrical conductivity of detonation nanodiamonds [8]. Hydrogenated detonation nanodiamonds in particular have attracted interest for their unusual interactions with water molecules [9], the creation of free radicals in water [10,11], use as radiosensitizers [12] and their fluorescence properties [13].

The two main routes towards the hydrogenation of DNDs are based on high-temperature annealing in molecular hydrogen gas $[8,14]$ and plasma-assisted modification [15]. Both make use of the high reactivity of hydrogen with carbon atoms on the diamond surface, but processing times, temperatures, experimental setup, and atmosphere differ significantly [16].

DNDs with various surface chemistries and different sizes show fluorescence [13,17-19], most of which appears to originate from non-diamond carbon rather than the diamond particle core [13], the latter being preferred for use in bioimaging applications for its better photostability [20]. However, it is known that DNDs can host fluorescent diamond lattice defects such as the nitrogen-vacancy (NV) center [21-23]. Our previous work has shown that among DNDs with several different surface modifications, hydrogenated DNDs overall exhibited spectral fluorescence properties that most closely resemble NV fluorescence [13], prompting further research to investigate the origin of this fluorescence.

Here, we fabricate hydrogenated DNDs via two different techniques using the same air oxidized DND starting material [24], as illustrated in Figure 1, and investigate their physico-chemical properties. Our two main aims were: (1) To identify potential differences in DND properties caused by the different processing techniques-including the degree of surface hydrogenation. (2) To identify whether the hydrogenated DND fluorescence mainly originates from non-diamond carbon or from fluorescent defects in the diamond lattice. We performed detailed material characterization using transmission electron microscopy-based electron energy loss spectroscopy (STEM-EELS), near-edge X-ray absorption fine structure (NEXAFS) spectroscopy, X-ray photoelectron emission spectroscopy (XPS), and Fourier-transform infrared (FTIR) absorption spectroscopy to determine the type and quantity of carbon bonds present in particles and the surface chemistry of both the starting material and the hydrogenated DND samples. We used dynamic light scattering (DLS) to assess the particles' colloidal properties and also performed fluorescence imaging and spectroscopy between room temperature and $10 \mathrm{~K}$ to investigate their fluorescence properties. Optical measurements at cryogenic temperatures are important, since they often allow for the clear identification of narrow spectral fluorescence features from defects inside the diamond lattice known as zero-phonon lines $[25,26]$.

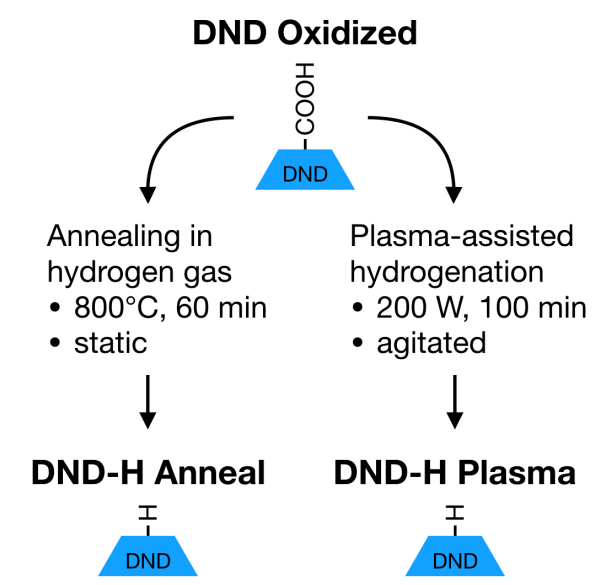

Figure 1. Schematic illustration of the two processes used for the hydrogenation of air oxidized detonation nanodiamonds (DND Oxidized) and the resulting particles (bottom). DND Oxidized particles were hydrogenated via annealing in pure hydrogen gas (DND-H Anneal) [8] and using a microwave plasma-assisted technique (DND-H Plasma) [16]. 


\section{Materials and Methods}

\subsection{Sample Preparation}

Detonation nanodiamonds (UD90, NanoBlox Inc., Boca Raton, FL, USA) were hydrogenated via annealing in hydrogen gas and through a microwave-assisted plasma treatment. Air oxidized $\left(425^{\circ} \mathrm{C}\right.$, $120 \mathrm{~min}$ ) particles [24] were used as a starting material for both processes.

Hydrogenation via annealing was performed by heating $250 \mathrm{mg}$ of the air oxidized DND powder in a high purity alumina $(100 \mathrm{~mm} \times 3 \mathrm{~mm} \times 18 \mathrm{~mm})$ combustion boat with a continued $50 \mathrm{sccm}$ flow of hydrogen gas in an alumina tube furnace (GSL-1800X-KS60 MTI corporation, Richmond, CA, USA) at $4{ }^{\circ} \mathrm{C} \mathrm{min}{ }^{-1}$ heating rate from room temperature up to $800{ }^{\circ} \mathrm{C}$ followed by $60 \mathrm{~min}$ at $800{ }^{\circ} \mathrm{C}$ in the flow of hydrogen. The tube was then cooled down to $\approx 50{ }^{\circ} \mathrm{C}$ at a rate of $4{ }^{\circ} \mathrm{C} \mathrm{min}-1$ under continued hydrogen flow prior to opening it and extracting the resulting DND-H Anneal material [8]. Typical yield of hydrogenated DND in these experiments was 69\% (173 mg).

Plasma-assisted hydrogenation was carried out using a home-made CVD reactor [15]. Briefly, DNDs deposited in quartz tube were exposed to a $\mathrm{H}_{2}$ microwave plasma with a gas pressure of $12 \mathrm{mbar}$, a gas flow of $10 \mathrm{sccm}$, and a microwave power of $200 \mathrm{~W}$ for $100 \mathrm{~min}$.

Throughout the manuscript, samples will be referred to as DND Oxidized (starting material), DND-H Anneal (annealed in hydrogen atmosphere), and DND-H Plasma (plasma-assisted hydrogenation).

\subsection{Material Characterization}

Fourier-transform infrared (FTIR) spectroscopy was carried out on functionalized particle powders using a PerkinElmer Frontier NIR spectrometer with a diamond crystal attenuated total reflectance (ATR) attachment. All other characterization experiments were performed on particles suspended in deionized (DI) water or on particles deposited on different substrates from aqueous suspension as described in the following.

Nanoparticles were suspended in DI water $\left(1 \mathrm{mg} \mathrm{mL}^{-1}\right)$ and sonicated for $1 \mathrm{~h}(66 \%$ duty cycle) in an ice bath with a horn sonicator (125 W ultrasound power). The resulting nanoparticle suspensions were centrifuged $(1000 \times g, 5 \mathrm{~min})$ to remove larger aggregates. The supernatant was used for further experiments and the nanoparticle concentration of every samples was estimated to be $\approx 0.4 \mathrm{mg} \mathrm{mL}^{-1}$ gravimetrically.

Scanning transmission electron microscopy (STEM) images and electron energy loss spectroscopy (EELS) data were acquired from dried nanoparticle suspensions $(10 \mu \mathrm{L})$ drop-cast onto silicon nitride TEM grids (SN100-A10Q33, SIMPore Inc., West Henrietta, NY, USA) using a JEOL 2100F operating at $80 \mathrm{keV}$ electron accelerating voltage.

X-ray photoelectron spectroscopy (XPS) data, collected with a photon energy of $850 \mathrm{eV}$, and near-edge X-ray absorption fine structure (NEXAFS) spectra between 270 and $320 \mathrm{eV}$, were obtained at the Australian Synchrotron, ANSTO on the soft X-ray beamline [27]. Nanoparticle suspensions were drop-cast onto commercially available Au-coated silicon wafers (646687, Sigma-Aldrich, St. Louis, MO USA). XPS spectra were corrected for sample charging during data acquisition with the use of a flood-gun and by later setting the C1s bulk line to $284.5 \mathrm{eV}$. Surface-sensitive NEXAFS spectra were acquired at an X-ray incidence angle of $55^{\circ}$ using partial electron yield (PEY) mode, with $243 \mathrm{eV}$ retarding voltage. The recorded signal was normalized by the "stable monitor method" [28], with intensity further normalized by setting the pre-edge intensity to 0 and the post edge to 1 . NEXAFS data were energy calibrated using a highly-oriented pyrolytic graphite (HOPG) reference and analyzed with QANT [29]. See SI for more details on XPS and NEXAFS data analysis.

Dynamic light scattering (DLS) data were obtained with a Malvern ZetaSizer Nano ZS using backscatter geometry. Size distributions and $\zeta$-potential distributions represent averages over 5 and 10 consecutive measurements respectively. 


\subsection{Optical Characterization}

In-solution fluorescence spectroscopy was conducted using a custom-built setup (see SI Figure S8 for details). Briefly, nanoparticle suspensions were excited in a cuvette (Type 18FL Micro, FireflySci, Staten Island, NY, USA) using weakly focused laser excitation (WhiteLaseSC 400, Fianium/NKT, UK). The excitation light was removed using optical long-pass filters (OD4, Edmund Optics, Barrington, NJ, USA) and the fluorescence signal analysed using a spectrometer (SpectraPro, Princeton Instruments, Trenton, NJ, USA) fitted with a CCD camera (PIXIS, Princeton Instruments, Trenton, NJ, USA). Pure DI water and silica nanoparticles suspended in DI water were used as a reference to remove the water Raman signal and potential scattering artefacts, respectively.

Fluorescence microscopy and spectroscopy experiments were conducted using a custom-built confocal fluorescence setup described in more detail elsewhere [30]. Briefly, a picosecond pulsed white light laser (WhiteLase SC400-8, Fianium/NKT Photonics, UK) operating at $532 \mathrm{~nm}, 5 \mathrm{MHz}$ repetition rate, and $50 \mu \mathrm{W}$ total beam power was used for excitation using a $532 \mathrm{~nm}$ laser line filter (Semrock, West Henrietta, NY, USA) and a $100 \times$ air objective with numerical aperture (NA) 0.9. The fluorescence signal was separated from the excitation beam using $532 \mathrm{~nm}$ dichroic and notch optical filters (both Semrock, USA). Avalanche photodiode detectors (SPCM-AQRH-14, Excelitas Technologies, Fremont, CA, USA) were employed for fluorescence detection and images were acquired using a piezo scanning stage (PInanoXYZ, PhysikInstrumente, Karlsruhe, Germany). A spectrometer (SpectraPro with a PIXIS CCD camera, Princeton Instruments, Trenton, NJ, USA) was used to obtain fluorescence spectra at selected locations in the image. A correlator card (TimeHarp 260, Picoquant, Berlin, Germany) was used to obtain time-resolved direct fluorescence decay traces. Cryogenic measurements were carried out in a liquid helium cryostat (Cryostation, Montana Instruments, Bozeman, MT, USA) using the setup described above, but using continuous wave $532 \mathrm{~nm}$ excitation $(100 \mu \mathrm{W})$ and a $50 \times$ air objective (NA 0.65). Nanoparticle suspensions $(20 \mu \mathrm{L})$ were drop-cast onto silicon wafer substrates and dried on a hot plate $\left(100{ }^{\circ} \mathrm{C}\right)$ in ambient air. Commercially available, $\approx 100 \mathrm{~nm}$ sized HPHT fluorescent nanodiamonds (Adamas Nanotechnologies, Raleigh, USA) were used as a reference material.

\section{Results}

\subsection{Physico-Chemical Properties}

\subsubsection{STEM-EELS}

Air oxidized DNDs were used as starting material and hydrogenated using two different processing routes, as illustrated in Figure 1. The first approach was based on high-temperature annealing in a pure hydrogen atmosphere (DND-H Anneal) [8], while the second approach was a plasma-assisted technique (DND-H Plasma) [15] (see Methods section for details). While the DND Oxidized starting material is known to contain less than 5\% non-diamond carbon [24], both hydrogenation techniques can lead to graphitization of the particles due to elevated temperatures of the processes. To identify differences in the degree of graphitization for the two hydrogenation techniques, we performed STEM and EELS experiments at $80 \mathrm{keV}$ accelerating voltage (using low acceleration voltage is important to minimize electron beam induced in situ graphitization of DND particles). STEM images of DND-H Anneal and DND-H Plasma samples are presented in Figure 2A,B, respectively. Both images show primary particles below $10 \mathrm{~nm}$ in diameter, all of which are part of larger aggregates of particles. Figure 2C,D show EELS maps for the regions in panels A and B. An EELS spectrum was acquired at each pixel, and the $\pi^{*}$ and $\sigma$ features were used to determine the $\mathrm{sp}^{2} / \mathrm{sp}^{3}$ ratio which is represented as a map of the percentage of $\mathrm{sp}^{2}$-hybridized carbon content (see SI for details).

We observed significant local variations in the $\mathrm{sp}^{2}$ content from $<10 \%$ to $>90 \%$. Regions with low amounts of $\mathrm{sp}^{2}$-hybridized carbon below $20 \%$, and hence relatively pure $\mathrm{sp}^{3}$-hybridized diamond, tended to be in the center of particles and particle aggregates. Conversely, the highest $\mathrm{sp}^{2}$-hybridized 
carbon signals above $90 \%$ were predominantly found along the edges of the agglomerates. This is consistent with the current understanding of the DND graphitization process, which occurs from the surface towards the center of the particle [31]. This significant difference in local carbon bonding characteristics also shows that the determination of a global average $\mathrm{sp}^{2}$ and $\mathrm{sp}^{3}$ values can be problematic. Nonetheless, the average $\mathrm{sp}^{2}$-hybridised carbon content for DND-H Anneal and DND-H Plasma were determined to be $39 \pm 19 \%$ and $32 \pm 17 \%$ respectively, where the error represents the standard deviation between individual pixels on the maps and quantifies the local variation in material properties visually evident in Figure 2C,D. Based on these results, we did not find a significant difference between the degree of graphitization induced by the two different functionalization techniques.
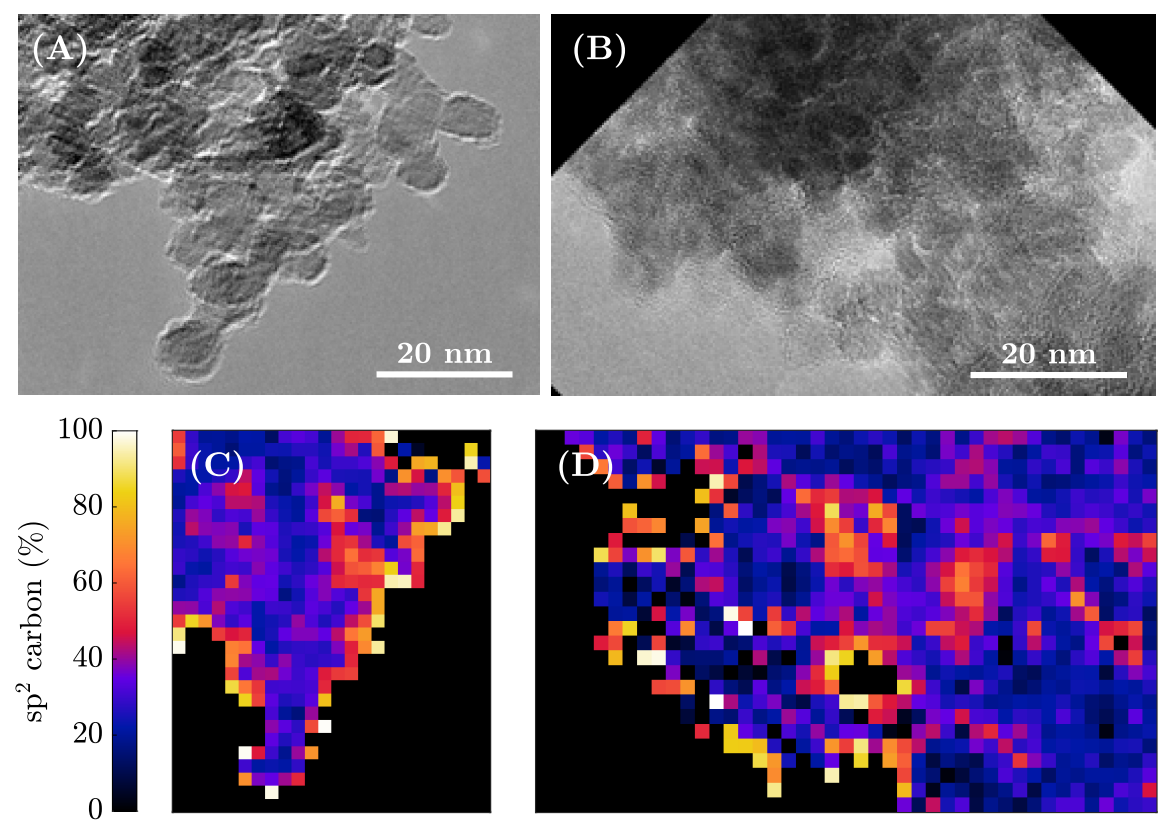

Figure 2. STEM images of $(\mathbf{A}) \mathrm{H}_{2}$ gas annealed and $(\mathbf{B}) \mathrm{H}_{2}$ plasma treated DND particles and EELS maps $(\mathbf{C}, \mathbf{D})$ of the same regions showing the $\mathrm{sp}^{2} / \mathrm{sp}^{3}$ fraction as a percentage of $\mathrm{sp}^{2}$-hybrydized carbon.

The image background is black. Measurements were performed at $80 \mathrm{keV}$ accelerating voltage.

\subsubsection{NEXAFS, XPS, and FTIR}

To investigate the surface chemistries and compositions of the DND samples in more detail, NEXAFS, XPS, and FTIR experiments were conducted, and the results are summarized in Figure 3. It is noteworthy that these measurements were performed on ensembles of particles. NEXAFS spectra (Figure $3 \mathrm{~A}$ ) show that the characteristic $2^{\text {nd }}$ diamond band gap at $302.4 \mathrm{eV}$ is present in all materials, indicating that a large fraction of the carbon atoms in the particles are indeed in the form of diamond $[32,33]$. The PEY NEXAFS data shown here originate mainly from the particle surface to a depth of $\approx 1-2 \mathrm{~nm}[34,35]$, suggesting that the starting material (DND Oxidized) as well as the two hydrogenated samples show strong diamond signatures close to the particles' surface. The pre-edge feature at $285.0 \mathrm{eV}$ (Figure 3B) is attributed to $\pi-\pi^{*}$ transitions in $\mathrm{sp}^{2}$-hybridized carbon [36] and is noticeably larger in the hydrogenated materials. This is an indication that both hydrogenation techniques resulted in partial graphitization of the ND surface-in agreement with the STEM-EELS results discussed above (Figure 2).

To quantify the extent of graphitization, we estimated the $\mathrm{sp}^{2} / \mathrm{sp}^{3}$ carbon ratio by integrating the area under the curves in Figure 3A between 283.0-285.5 eV $\left(\pi^{*}\right)$ and 298.0-320.0 eV $(\sigma)$ for each DND sample and the HOPG reference (see SI for details) [37]. We found that DND Oxidized has $\mathrm{sp}^{2}$ carbon content of $\approx 4 \%$, in good agreement with previous reports $[13,24]$. The amount of $\mathrm{sp}^{2}$ carbon 
increases to $\approx 19 \%$ in both DND-H Anneal and DND-H Plasma, which is in qualitative agreement with the average results obtained from EELS experiments (Figure 2).

We detected the emergence of a small peak at $282.3 \mathrm{eV}$ which did not exist in the oxidized starting material and appeared in both hydrogenated samples. This feature has previously been attributed to dangling bonds in partially hydrogenated nanodiamonds prepared under ultra-high vacuum [38]. Assuming this assignment is correct, it is worth noting that in the case of the hydrogenated DND samples studied here, the dangling bonds appear to be stable even after exposure to ambient atmospheric conditions. Regardless, the location and formation mechanism of the dangling bonds in our samples remain poorly understood.

All three materials show different features in the so-called "pre-edge region" between 286 and $288 \mathrm{eV}$. Surface chemistry-specific absorption features attributed to $\mathrm{C}=\mathrm{O}$ bonds at $286.5 \mathrm{eV}[39,40]$ and $\mathrm{C}-\mathrm{H}$ bonds at $\approx 287.3 \mathrm{eV}[41,42]$ are present in this region. Both hydrogenated samples show an increase in $\mathrm{C}-\mathrm{H}$ resonance compared to the oxidized starting material, indicating successful surface modification by both techniques. However, this effect appears to be more pronounced for DND-H Anneal, which shows a slightly stronger $\mathrm{C}-\mathrm{H}$ bond signal compared to DND-H Plasma. DND-H Plasma also has a more pronounced $\mathrm{C}=\mathrm{O}$ resonance, suggesting that more oxygen containing functional groups remain on this sample. However, due to the close spectral proximity of the $\mathrm{C}=\mathrm{O}$ and $\mathrm{C}-\mathrm{H}$ features [34], the differences are rather qualitative.

XPS spectra for all materials are shown in Figure 3C. All samples exhibit the characteristic C1s peak at $284.5 \mathrm{eV}$ and varying levels of $\mathrm{O} 1 \mathrm{~s}$ at $531.0 \mathrm{eV}$ [43]. The atomic concentration of oxygen decreases from 22 at\% pre-hydrogenation to 2 at\% (DND-H Anneal) and 6 at\% (DND-H Plasma) post-hydrogenation. Once again this shows successful partial removal of oxygen in both materials. In agreement with the NEXAFS results, DND-H Anneal appears to contain less oxygen than the DND-H Plasma sample. However, at least part of this oxygen could be associated with water molecules adsorbed on the DND surface and is not necessarily chemically bound to the surface $[9,44,45]$.
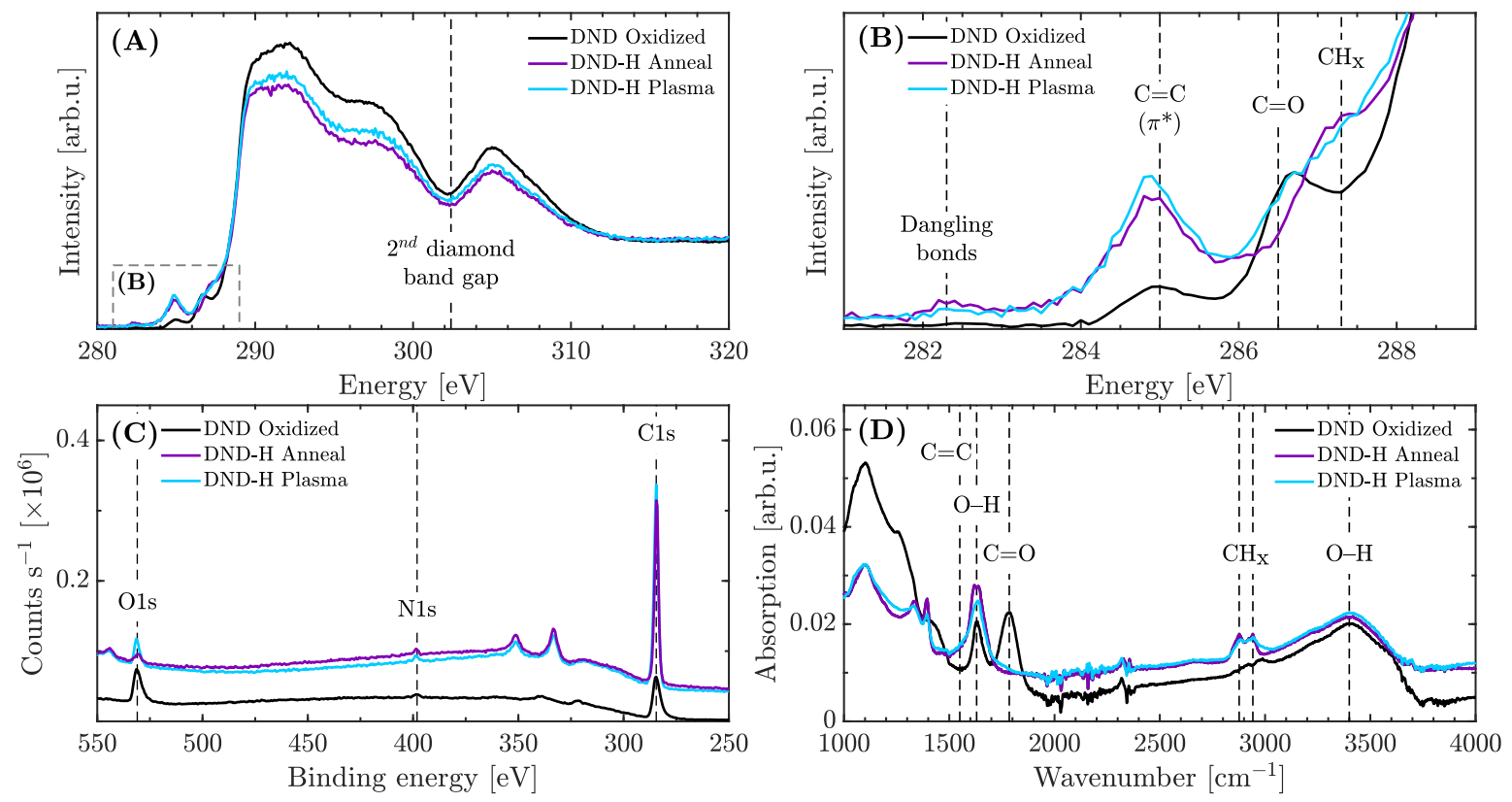

Figure 3. Spectroscopic characterization of hydrogenated DND materials and the oxidized starting material. (A) NEXAFS spectra of carbon K-edge; (B) carbon K-edge spectra displayed between 281 and $289 \mathrm{eV}$ to highlight pre-edge features; (C) XPS survey; and (D) FTIR spectra. Features of interest are marked with dashed lines.

All samples show a small N1s peak at $398.1 \mathrm{eV}$, indicating the presence of $\approx 1-2$ at $\%$ nitrogen in all samples. This consistency suggests that the nitrogen is embedded in the core of the DND 
particles, rather than being in the form of surface functional groups. This is feasible since DND particles are known to incorporate nitrogen on the order of 1 at $\%$ due to detonation synthesis from nitrogen-containing explosives [46-48].

FTIR spectra (Figure 3D) also qualitatively suggest the successful hydrogenation of both DND-H Anneal and DND-H Plasma samples: the $\mathrm{C}=\mathrm{O}$ peak at $1750 \mathrm{~cm}^{-1}$ disappears in both hydrogenated samples, while two $\mathrm{CH}_{x}$ stretch peaks appear at $2875 \mathrm{~cm}^{-1} \& 2930 \mathrm{~cm}^{-1}$, which are characteristic of hydrogen termination $[8,49,50]$. Both $\mathrm{O}-\mathrm{H}$ bending and stretching modes at $1630 \mathrm{~cm}^{-1}$ and $3400 \mathrm{~cm}^{-1}$ may indicate incomplete surface modification or be caused by adsorbed water molecules on the surface of the DNDs $[9,44,45]$. Another change due to hydrogenation is the appearance of a broad shoulder at $\approx 1550 \mathrm{~cm}^{-1}$ which is typically associated with $\mathrm{C}=\mathrm{C}$ stretching $[44,51,52]$.

\subsection{Colloidal Properties}

The hydrogenated DND particles were dispersed in DI water to study their colloidal and optical properties in suspension. Briefly, DND powder was suspended in DI water, sonicated for $1 \mathrm{~h}(125 \mathrm{~W}$, $66 \%$ duty cycle), centrifuged $(1000 \times g, 5 \mathrm{~min})$, and the supernatant used for experiments. The resulting particle concentration was estimated to be $\approx 0.4 \mathrm{mg} \mathrm{mL}^{-1}$ for both DND-H Anneal and DND-H Plasma (see Methods section for details).

Dynamic light scattering (DLS) was used to investigate the size and $\zeta$-potential of DND aggregates in suspension. Both DND-H Anneal and DND-H Plasma showed a polydisperse size distribution with particle diameters from $\approx 20 \mathrm{~nm}$ to well above $100 \mathrm{~nm}$ (see SI Figure S6 for size distributions). Smaller aggregates contribute more to the overall size distribution in the DND-H Anneal sample compared to the DND-H Plasma sample. The DND-H Anneal sample exhibited a very high $\zeta$-potential of about $+70 \mathrm{mV}$, slightly higher than the value of $+50 \mathrm{mV}$ obtained for DND-H Plasma (see SI for more details). One possible explanation for the difference in the observed $\zeta$-potential could be the slightly higher density of remaining oxygen containing surface groups in the DND-H Plasma sample, which often carry a negative charge in water [13]. The DLS results as well as the quantification of $\mathrm{sp}^{2}$-bonded carbon in both samples are summarized in Table 1.

Table 1. Summary of material properties of the hydrogenated DND particles, including $\zeta$-potential, aggregate diameter, and $\mathrm{sp}^{2}$ content determined using NEXAFS.

\begin{tabular}{lccc}
\hline Sample & Aggregate Diameter $(\mathbf{n m})$ & $\zeta$-Potential $(\mathbf{m V})$ & $\mathbf{s p}^{2}$-Bonded Carbon $\mathbf{( \% )}$ \\
\hline DND-H Anneal & $\approx 20-200$ & $72 \pm 7$ & 19 \\
DND-H Plasma & $\approx 20-200$ & $53 \pm 10$ & 19 \\
\hline
\end{tabular}

\subsection{Fluorescence Properties}

\subsubsection{In-Solution Fluorescence Spectroscopy}

The optical properties of both hydrogenated DND samples suspended in water were investigated using absorption and fluorescence spectroscopy. Light scattering by nanodiamonds in the $20-100 \mathrm{~nm}$ size range is significant [53]. Hence, an absorption spectrometer fitted with an integrating sphere was used to remove this scattering contribution and acquire the absorption spectra shown in Figure 4A. For both samples light absorption is most pronounced in the UV and blue parts of the spectrum and monotonically decreases towards the red and near-infrared spectral regions. It is interesting to note that light is still weakly absorbed between 700 and $800 \mathrm{~nm}$. These observations are in general agreement with previous reports for DNDs with different functionalizations $[18,54,55]$.

Both hydrogenated DND samples demonstrate broad and weak red fluorescence upon $450 \mathrm{~nm}$ light excitation, as shown in Figure 4B, where the water Raman signal was removed for clarity (see SI Figure S9 for raw data). Both samples also show fluorescence when excited with light at longer wavelengths, but in both cases the relative fluorescence brightness decreases monotonically. In the spectral region investigated, DND-H Anneal and DND-H Plasma are most efficiently excited at 
$\approx 450 \mathrm{~nm}$ and $400 \mathrm{~nm}$, respectively, though DND-H Plasma may have an excitation maximum farther in the UV spectral region beyond our detection limit. In agreement with our previous work, we found only a weak excitation wavelength dependence on the fluorescence peak position [13] (see SI Figure S9 for spectra). Overall, DND-H Anneal absorbs and emits $\approx 31 \%-33 \%$ more light at $450 \mathrm{~nm}$ compared to the DND-H Plasma sample, suggesting that the fluorescence quantum yield is similar for both samples.
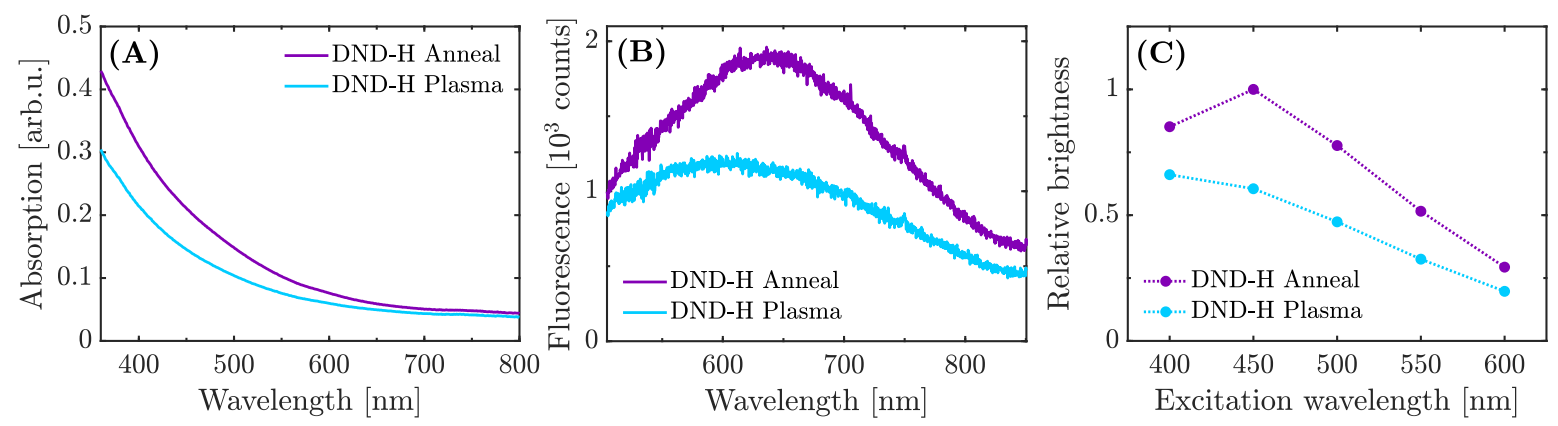

Figure 4. Optical properties of hydrogenated DND particles suspended in water $\left(0.4 \mathrm{mg} \mathrm{mL}^{-1}\right)$. (A) Absorption spectra; (B) fluorescence spectra for $450 \mathrm{~nm}$ excitation wavelength $\lambda_{\text {ex }}$; (C) fluorescence brightness (integrated between 660 and $800 \mathrm{~nm}$ for each $\lambda_{\text {ex }}$, detailed in SI) as a function of excitation wavelengths ranging from 400 to $600 \mathrm{~nm}$.

\subsubsection{Solid-State Fluorescence Spectroscopy}

The two hydrogenated DND samples were also investigated in a dried state dispersed on a silicon wafer substrate using a custom-built confocal fluorescence imaging and spectroscopy system. Figure 5 shows the fluorescence spectra (Figure 5A), time-resolved fluorescence decay traces (Figure 5B), and photostability traces (Figure 5C) (see SI Figure S10 for fluorescence maps). The data in Figure 5 represent averages over 10 randomly selected spots, while the individual spectra can be found in SI Figure S11. In agreement with the spectral fluorescence characteristics observed for particles in water, both DND samples show broad red fluorescence between 550 and $800 \mathrm{~nm}$. The width of the fluorescence spectrum of particles dispersed on a substrate is narrower than for particles suspended in water (full width at half maximum of $160 \mathrm{~nm}$ compared to $>240 \mathrm{~nm}$ in water).

Both samples show similar fluorescence decay characteristics (Figure 5B), which are dominated by a fast decay component of $<5 \mathrm{~ns}$ and a slower one $>25 \mathrm{~ns}$. Ninety-nine percent of photons are emitted within the first $5 \mathrm{~ns}$ of the decay, and a precise lifetime cannot be determined as the fluorescence decay approaches the time resolution of the system shown by the instrument response function (IRF).
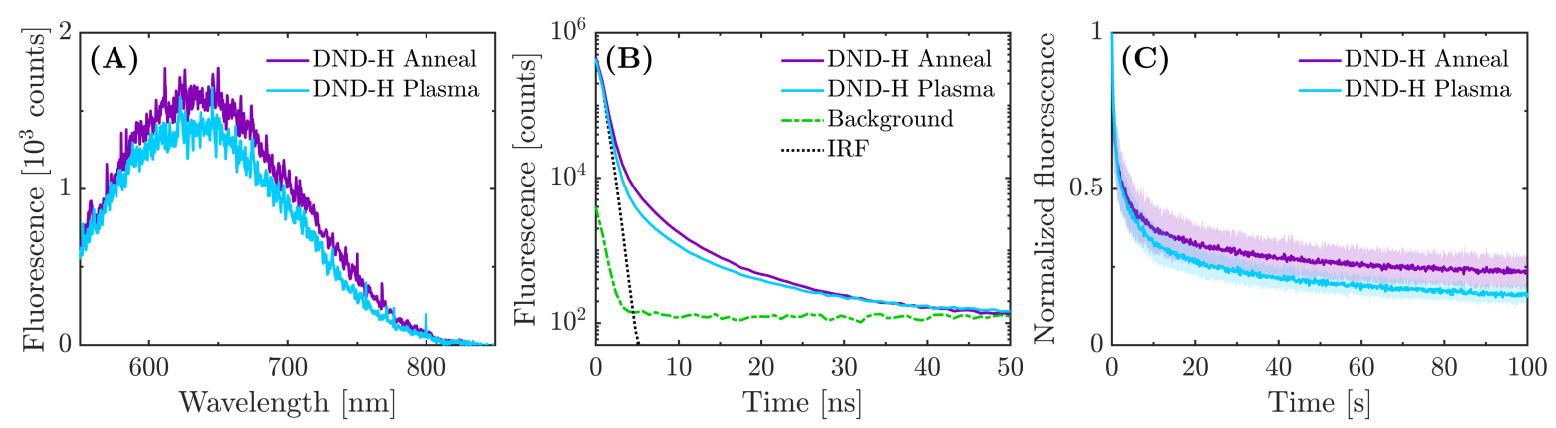

Figure 5. Solid state fluorescence properties of hydrogenated DND particles dispersed on a silicon wafer substrate. (A) Fluorescence spectra; (B) time-resolved fluorescence decay traces, including background signal and instrument response function (IRF); (C) normalized fluorescence intensity as a function of time. Particles were excited with $532 \mathrm{~nm}$ pulsed laser light (5 MHz repetition rate, $50 \mu \mathrm{W}$ average power) and all fluorescence above $540 \mathrm{~nm}$ was collected. Each trace represents an average over 10 individual spots. The shaded area in $(\mathbf{C})$ represents the standard deviation between individual measurements. 
Under continuous excitation for $100 \mathrm{~s}$ both samples show significant photobleaching during the first $20 \mathrm{~s}$ of illumination and then stabilize at or above $20 \%$ of the initial fluorescence intensity (Figure 5C). Interestingly, we found that all particles remain partially fluorescent even after extended illumination times, which contrasts with the behavior of organic fluorophores [56]. We also found that the partial photobleaching does not change the spectral fluorescence characteristics. However, since the exact molecular or atomic origin of the fluorescence observed here remains unknown, the mechanism underlying the photobleaching process cannot be identified based on our findings.

\subsubsection{Cryogenic Fluorescence Spectroscopy}

Fluorescence spectroscopy at cryogenic temperatures was conducted with the aim of better understanding the origins of the observed fluorescence from hydrogenated DND. Figure 6 shows fluorescence spectra for both samples at room temperature (293 K) and $10 \mathrm{~K}$ (see SI Figure S12 for spectra at intermediate temperatures). The fluorescence spectra of both samples only show an insignificant temperature dependence. In both cases a weak, $\approx 30 \mathrm{~nm}$ blue-shift in the fluorescence spectrum occurs upon the evacuation of the cryogenic sample chamber (see Figure 6A,B, black trace in ambient air and red trace in vacuum), suggesting that the surface-adsorbed molecules influence the fluorescence process. We did not observe any clear zero-phonon lines, which are typical for fluorescent defects located in the diamond crystal lattice. However, our experiments show that single-crystal high-pressure high-temperature nanodiamonds containing NV centers do not necessarily exhibit zero-phonon lines (unpublished data). Other reports show that high concentrations of $\mathrm{N}$ atoms near $\mathrm{NV}$ centers can lead to a broadening of the NV zero-phonon lines [26]. Hence, the absence of zero-phonon lines is not in contradiction with a potential fluorescence origin from diamond lattice defects.

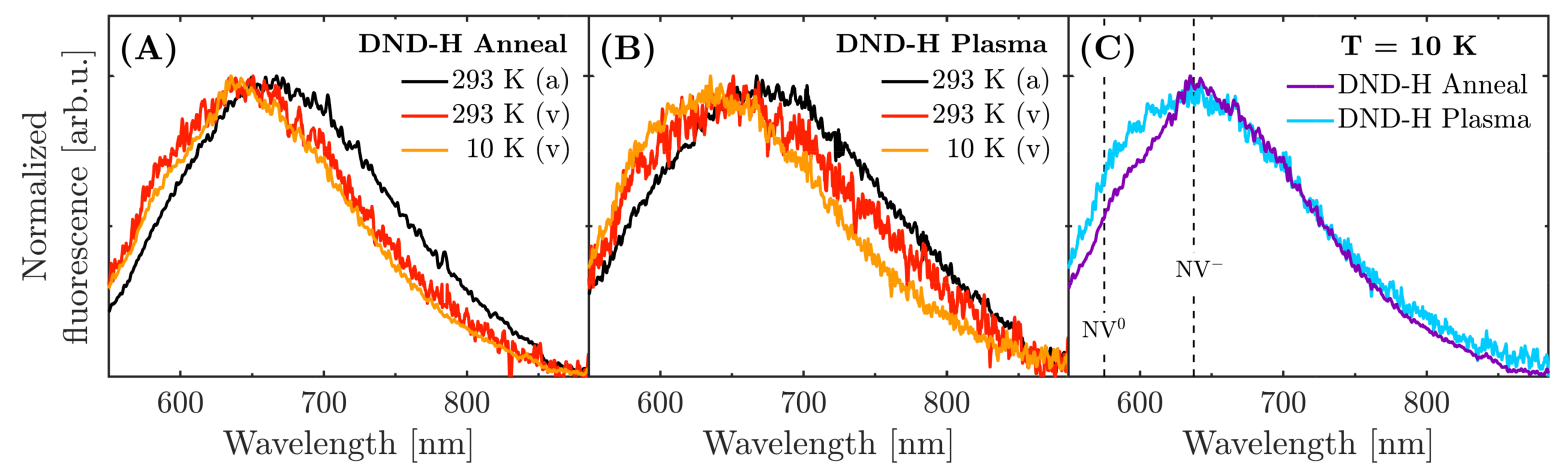

Figure 6. Temperature dependence of the fluorescence spectra of hydrogenated DND particles obtained using $532 \mathrm{~nm}$ excitation. Fluorescence spectra of (A) DND-H Anneal and (B) DND-H Plasma samples at temperatures $293 \mathrm{~K}$ and $10 \mathrm{~K}$ (in ambient air "a" and vacuum "v"). (C) Comparison of DND-H Anneal and DND-H Plasma samples at $10 \mathrm{~K}$. The known spectral positions of the NV center zero-phonon lines are marked by dashed lines.

\section{Discussion}

Our material characterization results are in agreement with previous studies on DND-H produced via annealing [8] and the plasma-assisted approach [15]. Our results extend existing data through the use of nanoscale EELS experiments in combination with NEXAFS and XPS and allow for a direct comparison between the two surface modification techniques. EELS and NEXAFS experiments suggest that both hydrogenation techniques induce partial graphitization of the oxidized starting material and increase the amount of $\mathrm{sp}^{2}$-hybridized carbon from $4 \%$ in the starting material to between $20 \%$ and $30 \%$ in the hydrogenated samples. EELS maps acquired using STEM also highlight the local variation in the $\mathrm{sp}^{2} / \mathrm{sp}^{3}$ carbon ratio at the nanoscale. At the same time, a clear $2^{\text {nd }}$ diamond band gap in NEXAFS spectra of both samples shows that both hydrogenation techniques preserve $\mathrm{sp}^{3}$ carbon bonds to a large extent. 
Our study of the fluorescence properties focuses on the type of fluorescence exhibited by the vast majority of particles, rather than the fluorescence from a small subset of particles that can be clearly attributed to the NV center. In this sense it extends our previous study [13], which aimed at identifying the origin of the fluorescence that cannot be immediately attributed to NV centers.

DND Oxidized shows extremely weak fluorescence that is barely detectable, for example, for particles suspended in water [13]. Hence, the fluorescence observed here must be caused by the hydrogenation process. The hydrogenation process itself, i.e., the replacement of other surface groups such as carboxyl and hydroxyl groups by $\mathrm{C}-\mathrm{H}_{x}$ bonds, can hardly produce any fluorescent moieties. Hence, two other possible mechanisms remain: First, the "activation" of NV centers as a result of the surface modification. It has been estimated that NV centers exist in DNDs on the order of $100 \mathrm{ppm}$ [57], most of which are not optically active. It is also known that the diamond surface properties of bulk diamond have a strong effect on the optical properties of near-surface NV centers [58,59]. It is therefore conceivable that the hydrogenation activates previously inactive NV centers in the DND particle core. However, our results do not support this hypothesis, since none of the characteristic properties of NV centers in diamond, such as zero-phonon lines at $575 \mathrm{~nm}$ and $638 \mathrm{~nm}$, long (>10 ns) fluorescence lifetime, and extreme photostability were observed; not even at cryogenic temperatures, where zero-phonon line fluorescence is generally enhanced due to the suppression of phonons at low temperatures. There is also the possibility of hydrogen diffusion into the diamond core which could lead to the passivation of NV fluorescence [60].

The second possibility is that the fluorescence originates from the significant non-diamond carbon created on the surface of the DND particles as a result of the hydrogenation processes. It is known that various forms of $\mathrm{sp}^{2}$-hybridised carbon can give rise to fluorescence and that the fluorescence properties can vary greatly depending on the exact chemical composition of the material $[61,62]$. In the present study, the appearance of fluorescence in the DND-H materials coincides with a significant increase in non-diamond carbon compared to the largely non-fluorescent starting material. This suggests $\mathrm{sp}^{2}$ carbon in combination with other impurities such as nitrogen and oxygen as the likely sources of the observed fluorescence. However, our current results are insufficient to prove this hypothesis unambiguously.

\section{Conclusions}

Hydrogenated detonation nanodiamonds created via an annealing and a plasma-assisted approach from the same starting material exhibit very similar fluorescence and physico-chemical properties overall. Both approaches lead to successful hydrogenation of the DND surface. Annealing in pure hydrogen removes oxygen-containing groups at the particle surface more efficiently than the plasma-assisted hydrogenation, leading to a more positive and overall very high $\zeta$-potential $(+72 \mathrm{mV})$ of the DND Anneal sample in water suspension. Starting from $4 \%$ non-diamond $\mathrm{sp}^{2}$-hybridized carbon in the DND Oxidized sample, STEM-EELS and NEXAFS experiments suggest that both hydrogenation techniques lead to similar partial graphitization of particles and increase the non-diamond carbon content to $\approx 20 \%-30 \%$.

In agreement with the physico-chemical characterization, we also found the fluorescence properties of both samples to be largely similar. In aqueous suspension, both samples showed broad and relatively weak (on the same order of magnitude as the water Raman signal) fluorescence in the spectral region between 550 and $800 \mathrm{~nm}$, which is most efficiently excited at $450 \mathrm{~nm}$ (DND-H Anneal) and $400 \mathrm{~nm}$ (DND-H Plasma). This fluorescence is also present for both particle types when dried on a silicon wafer substrate and only shows a weak blue-shift of $\approx 30 \mathrm{~nm}$ when the temperature is reduced to $10 \mathrm{~K}$. However, even at cryogenic temperatures, zero-phonon lines of the NV center could not be clearly identified in our samples. While it is known that individual hydrogenated DND particles do exhibit NV fluorescence [13], our results suggest that the fluorescence emitted by the vast majority of particles originates from non-diamond carbon on the surface of the particles. 
Supplementary Materials: The following are available online at http://www.mdpi.com/2311-5629/6/1/7/s1. Figure S1: Electron-energy loss spectra of the C1s K-edge for DND-H Anneal and DND-H Plasma samples; Figure S2: Carbon K-edge NEXAFS spectra with shaded $\mathrm{sp}^{2}$ and $\mathrm{sp}^{3}$ ranges used for determining the $\mathrm{sp}^{2} / \mathrm{sp}^{3}$ rati0; Figure S3: Full XPS spectra of the starting oxidized DND and both hydrogenated DND samples. Spectra not corrected for charging effects; Figure S4: Full range of FTIR data for the starting DND Oxidized material and the two hydrogenated DND samples; Figure S5: Colloidal properties of hydrogenated DND particles dispersed in water at $\sim 0.4 \mathrm{mg} \mathrm{mL}^{-1}$. (A) The averaged intensity-weighted size distribution of the DND aggregates, and (B) their corresponding averaged z-potential; Figure S6: Raw dynamic light scattering results for both (A-C) DND-H Anneal and (D-F) DND-H Plasma samples with (A \& D) intensity; (B \& E) number; and (C \& F) volume weighted distributions; Figure S7: Raw z-potential for (A) DND-H Anneal and (B) DND-H Plasma samples; Figure S8: Schematic of our custom-built in-solution spectroscopy setup used for all in-solution experiments with all components labelled; Figure S9: In-solution spectra of (A) DND-H Anneal and (B) DND-H Plasma samples at different excitation wavelengths; normalized for differences in excitation power. (C) Change in peak emission wavelength as a function of excitation wavelength. Raw (D) DND-H Anneal and (E) DND-H Plasma results not corrected for differences in excitation power or $(\mathrm{F})$ water signal; Figure S10: Room-temperature solid state PL maps of (A) DND-H Anneal and (B) DND-H Plasma samples drop-cast onto Si wafers. Excited using a $532 \pm 30 \mathrm{~nm}$ pulsed excitation source at $5 \mathrm{MHz}$ repetition rate at $50 \mu \mathrm{W}$; Figure S11: Room-temperature solid state fluorescence spectra of (A) DND-H Anneal and (B) DND-H Plasma samples corresponding to PL maps in Figure S10; Figure S12: Averaged fluorescence spectra of (A) DND-H Anneal and (B) DND-H Plasma samples for temperatures ranging from $293 \mathrm{~K}$ in air atmosphere and vacuum to $10 \mathrm{~K}$. Faded envelopes around solid lines indicate standard deviation between measurements; Figure S13: Peak emission wavelength of hydrogenated DND samples on Si substrates as a function of changing temperature. Postscripts ' $a$ ' and ' $v$ ' refer to ambient atmospheric pressure and in-vacuum respectively. All measurements at temperatures $<293 \mathrm{~K}$ were performed in vacuum; Table S1: Combination of excitation wavelength, power, and filters used for in-solution fluorescence spectroscopy measurements. Total acquisition time for each spectrum was $100 \mathrm{~s}$. Excitation laser source was set to $80 \mathrm{MHz}$ repetition rate for all excitation conditions.

Author Contributions: Conceptualization, G.T., B.C.G., and P.R.; methodology, G.T., P.R., A.S., B.J.M., and A.T.; software, G.T.; validation, G.T.; formal analysis, G.T., B.J.M., and P.R.; investigation, G.T., A.S., N.D., B.J.M., E.M., L.T., and P.R.; resources, H.A.G., J.-C.A., I.M.A., and V.M.; data curation, G.T.; writing-original draft preparation, G.T. and P.R.; writing-review and editing, G.T., A.S., N.D., B.J.M., H.A.G., I.M.A., L.T., A.T., J.-C.A., V.N.M., B.C.G., and P.R.; visualization, G.T. and P.R.; supervision, B.C.G. and P.R.; project administration, P.R. and B.C.G.; funding acquisition, P.R. and B.C.G. All authors have read and agreed to the published version of the manuscript.

Funding: This work was supported by the Australian Research Council (ARC) through the Centre of Excellence for Nanoscale BioPhotonics (CE140100003) and Linkage Infrastructure, Equipment and Facilities grant (LE140100131). G.T. acknowledges funding through the RMIT PhD Scholarship. P.R. acknowledges funding through the RMIT Vice-Chancellor's Research Fellowship.

Acknowledgments: This work was performed in part at the RMIT Micro Nano Research Facility (MNRF) in the Victorian Node of the Australian National Fabrication Facility (ANFF). The authors acknowledge the use of the RMIT Microscopy and Microanalysis Facility (RMMF). This research was partly undertaken on the Soft X-Ray Spectroscopy beamline at the Australian Synchrotron, part of ANSTO.

Conflicts of Interest: The authors declare no conflict of interest. The funders had no role in the design of the study; in the collection, analyses, or interpretation of data; in the writing of the manuscript, or in the decision to publish the results.

\author{
Abbreviations \\ The following abbreviations are used in this manuscript: \\ DND Detonation nanodiamond \\ DND-H Hydrogenated detonation nanodiamond \\ HOPG Highly-oriented pyrolytic graphite \\ STEM Scanning transmission electron microscopy \\ EELS Electron energy loss spectroscopy \\ NEXAFS Near-edge $X$-ray absorption fine structure \\ PEY Partial electron yield \\ XPS X-ray photoelectron spectroscopy \\ FTIR Fourier-transform infrared \\ DLS Dynamic light scattering \\ NV Nitrogen vacancy \\ HPHT High-pressure high-temperature \\ DI Deionized
}




\section{References}

1. Mochalin, V.N.; Shenderova, O.; Ho, D.; Gogotsi, Y. The Properties and Applications of Nanodiamonds. Nat. Nanotechnol. 2012, 7, 11-23. [CrossRef]

2. Nunn, N.; Torelli, M.; McGuire, G.; Shenderova, O. Nanodiamond: A High Impact Nanomaterial. Curr. Opin. Solid State Mater. Sci. 2017, 21, 1-9. [CrossRef]

3. Lee, D.K.; Kee, T.; Liang, Z.; Hsiou, D.; Miya, D.; Wu, B.; Osawa, E.; Chow, E.K.H.; Sung, E.C.; Kang, M.K.; et al. Clinical Validation of a Nanodiamond-Embedded Thermoplastic Biomaterial. Proc. Natl. Acad. Sci. USA 2017, 114, E9445-E9454. [CrossRef] [PubMed]

4. Zhang, X.Q.; Lam, R.; Xu, X.; Chow, E.K.; Kim, H.J.; Ho, D. Multimodal Nanodiamond Drug Delivery Carriers for Selective Targeting, Imaging, and Enhanced Chemotherapeutic Efficacy. Adv. Mater. 2011, 23, 4770-4775. [CrossRef] [PubMed]

5. Williams, O.A.; Douhéret, O.; Daenen, M.; Haenen, K.; Ōsawa, E.; Takahashi, M. Enhanced Diamond Nucleation on Monodispersed Nanocrystalline Diamond. Chem. Phys. Lett. 2007, 445, 255-258. [CrossRef]

6. Berman, D.; Narayanan, B.; Cherukara, M.J.; Sankaranarayanan, S.K.R.S.; Erdemir, A.; Zinovev, A.; Sumant, A.V. Operando Tribochemical Formation of Onion-like-Carbon Leads to Macroscale Superlubricity. Nat. Commun. 2018, 9, 1-9. [CrossRef]

7. Pakes, C.I.; Garrido, J.A.; Kawarada, H. Diamond Surface Conductivity: Properties, Devices, and Sensors. MRS Bull. 2014, 39, 542-548. [CrossRef]

8. Kondo, T.; Neitzel, I.; Mochalin, V.N.; Urai, J.; Yuasa, M.; Gogotsi, Y. Electrical Conductivity of Thermally Hydrogenated Nanodiamond Powders. J. Appl. Phys. 2013, 113, 214307. [CrossRef]

9. Petit, T.; Puskar, L.; Dolenko, T.; Choudhury, S.; Ritter, E.; Burikov, S.; Laptinskiy, K.; Brzustowski, Q.; Schade, U.; Yuzawa, H.; et al. Unusual Water Hydrogen Bond Network around Hydrogenated Nanodiamonds. J. Phys. Chem. C 2017, 121, 5185-5194. [CrossRef]

10. Petit, T.; Arnault, J.C.; Girard, H.; Grall, R.; Chevillard, S.; Delic, J. Use of Nanodiamonds for Generating Free Radicals for Therapeutic Purposes under Radiation. U.S. Patent, US10,391,172B2, 27 August 2019.

11. Kurzyp, M.; A. Girard, H.; Cheref, Y.; Brun, E.; Sicard-Roselli, C.; Saada, S.; Arnault, J.C. Hydroxyl Radical Production Induced by Plasma Hydrogenated Nanodiamonds under X-Ray Irradiation. Chem. Commun. 2017, 53, 1237-1240. [CrossRef] [PubMed]

12. Grall, R.; Girard, H.; Saad, L.; Petit, T.; Gesset, C.; Combis-Schlumberger, M.; Paget, V.; Delic, J.; Arnault, J.C.; Chevillard, S. Impairing the Radioresistance of Cancer Cells by Hydrogenated Nanodiamonds. Biomaterials 2015, 61, 290-298. [CrossRef] [PubMed]

13. Reineck, P.; Lau, D.W.; Wilson, E.R.; Fox, K.; Field, M.R.; Deeleepojananan, C.; Mochalin, V.N.; Gibson, B.C. Effect of Surface Chemistry on the Fluorescence of Detonation Nanodiamonds. ACS Nano 2017, 11, 10924-10934. [CrossRef] [PubMed]

14. Mochalin, V.N.; Osswald, S.; Portet, C.; Yushin, G.; Hobson, C.; Havel, M.; Gogotsi, Y. High Temperature Functionalization and Surface Modification of Nanodiamond Powders. MRS Online Proc. Libr. Arch. 2007, 1039. [CrossRef]

15. Girard, H.; Arnault, J.; Perruchas, S.; Saada, S.; Gacoin, T.; Boilot, J.P.; Bergonzo, P. Hydrogenation of Nanodiamonds Using MPCVD: A New Route toward Organic Functionalization. Diam. Relat. Mater. 2010, 19, 1117-1123. [CrossRef]

16. Arnault, J.; Girard, H. Hydrogenated Nanodiamonds: Synthesis and Surface Properties. Curr. Opin. Solid State Mater. Sci. 2017, 21, 10-16. [CrossRef]

17. Wang, Z.; Xu, C.; Liu, C. Surface Modification and Intrinsic Green Fluorescence Emission of a Detonation Nanodiamond. J. Mater. Chem. C 2013, 1, 6630-6636, [CrossRef]

18. Reineck, P.; Lau, D.W.; Wilson, E.R.; Nunn, N.; Shenderova, O.A.; Gibson, B.C. Visible to Near-IR Fluorescence from Single-Digit Detonation Nanodiamonds: Excitation Wavelength and $\mathrm{pH}$ Dependence. Sci. Rep. 2018, 8, 2478. [CrossRef]

19. Vervald, A.M.; Burikov, S.A.; Shenderova, O.A.; Nunn, N.; Podkopaev, D.O.; Vlasov, I.I.; Dolenko, T.A. Relationship Between Fluorescent and Vibronic Properties of Detonation Nanodiamonds and Strength of Hydrogen Bonds in Suspensions. J. Phys. Chem. C 2016, 120, 19375-19383. [CrossRef] 
20. Turcheniuk, K.; Mochalin, V.N. Biomedical Applications of Nanodiamond (Review). Nanotechnology 2017, 28, 252001. [CrossRef]

21. Bradac, C.; Gaebel, T.; Naidoo, N.; Sellars, M.; Twamley, J.; Brown, L.; Barnard, A.; Plakhotnik, T.; Zvyagin, A.; Rabeau, J. Observation and Control of Blinking Nitrogen-Vacancy Centres in Discrete Nanodiamonds. Nat. Nanotechnol. 2010, 5, 345. [CrossRef]

22. Reineck, P.; Capelli, M.; Lau, D.; Jeske, J.; Field, M.; Ohshima, T.; Greentree, A.; Gibson, B. Bright and Photostable Nitrogen-Vacancy Fluorescence from Unprocessed Detonation Nanodiamond. Nanoscale 2017, 9, 497-502. [CrossRef] [PubMed]

23. Terada, D.; Segawa, T.F.; Shames, A.I.; Onoda, S.; Ohshima, T.; Ōsawa, E.; Igarashi, R.; Shirakawa, M. Monodisperse Five-Nanometer-Sized Detonation Nanodiamonds Enriched in Nitrogen-Vacancy Centers. ACS Nano 2019, 13, 6461-6468. [CrossRef] [PubMed]

24. Osswald, S.; Yushin, G.; Mochalin, V.; Kucheyev, S.O.; Gogotsi, Y. Control $\mathrm{of} \mathrm{sp}^{2} / \mathrm{sp}^{3}$ Carbon Ratio and Surface Chemistry of Nanodiamond Powders by Selective Oxidation in Air. J. Am. Chem. Soc. 2006, 128, 11635-11642. [CrossRef] [PubMed]

25. Zhao, H.Q.; Fujiwara, M.; Okano, M.; Takeuchi, S. Observation of 1.2-GHz Linewidth of Zero-Phonon-Line in Photoluminescence Spectra of Nitrogen Vacancy Centers in Nanodiamonds Using a Fabry-Perot Interferometer. Opt. Express OE 2013, 21, 29679-29686. [CrossRef]

26. Manson, N.B.; Hedges, M.; Barson, M.S.J.; Ahlefeldt, R.; Doherty, M.W.; Abe, H.; Ohshima, T.; Sellars, M.J. $\mathrm{NV}^{-}-\mathrm{N}^{+}$Pair Centre in 1b Diamond. New J. Phys. 2018, 20, 113037. [CrossRef]

27. Cowie, B.C.C.; Tadich, A.; Thomsen, L. The Current Performance of the Wide Range (90-2500 eV) Soft X-ray Beamline at the Australian Synchrotron. AIP Conf. Proc. 2010, 1234, 307-310. [CrossRef]

28. Watts, B.; Thomsen, L.; Dastoor, P.C. Methods in Carbon K-Edge NEXAFS: Experiment and Analysis. J. Electron Spectrosc. Relat. Phenom. 2006, 151, 105-120. [CrossRef]

29. Gann, E.; McNeill, C.R.; Tadich, A.; Cowie, B.C.C.; Thomsen, L. Quick AS NEXAFS Tool (QANT): A Program for NEXAFS Loading and Analysis Developed at the Australian Synchrotron. J. Synchrotron Radiat. 2016, 23, 374-380. [CrossRef]

30. Reineck, P.; Trindade, L.F.; Havlik, J.; Stursa, J.; Heffernan, A.; Elbourne, A.; Orth, A.; Capelli, M.; Cigler, P.; Simpson, D.A.; et al. Not All Fluorescent Nanodiamonds Are Created Equal: A Comparative Study. Part. Part. Syst. Charact. 2019, 36, 1900009. [CrossRef]

31. Xu, N.S.; Chen, J.; Deng, S.Z. Effect of Heat Treatment on the Properties of Nano-Diamond under Oxygen and Argon Ambient. Diam. Relat. Mater. 2002, 11, 249-256. [CrossRef]

32. Gruen, D.M.; Krauss, A.R.; Zuiker, C.D.; Csencsits, R.; Terminello, L.J.; Carlisle, J.A.; Jimenez, I.; Sutherland, D.G.J.; Shuh, D.K.; Tong, W.; et al. Characterization of Nanocrystalline Diamond Films by Core-level Photoabsorption. Appl. Phys. Lett. 1996, 68, 1640-1642. [CrossRef]

33. Morar, J.F.; Himpsel, F.J.; Hollinger, G.; Hughes, G.; Jordan, J.L. Observation of a C1s Core Exciton in Diamond. Phys. Rev. Lett. 1985, 54, 1960-1963. [CrossRef] [PubMed]

34. Shpilman, Z.; Gouzman, I.; Minton, T.K.; Shen, L.; Stacey, A.; Orwa, J.; Prawer, S.; Cowie, B.C.C.; Hoffman, A. A near Edge X-Ray Absorption Fine Structure Study of Oxidized Single Crystal and Polycrystalline Diamond Surfaces. Diam. Relat. Mater. 2014, 45, 20-27. [CrossRef]

35. Stacey, A.; Cowie, B.C.C.; Orwa, J.; Prawer, S.; Hoffman, A. Diamond C1s Core-Level Excitons: Surface Sensitivity. Phys. Rev. B 2010, 82, 125427. [CrossRef]

36. Stöhr, J. NEXAFS Spectroscopy; Springer: Berlin/Heidelberg, Germany, 1992.

37. Fallon, P.J.; Veerasamy, V.S.; Davis, C.A.; Robertson, J.; Amaratunga, G.A.J.; Milne, W.I.; Koskinen, J. Properties of Filtered-Ion-Beam-Deposited Diamondlike Carbon as a Function of Ion Energy. Phys. Rev. B 1993, 48, 4777-4782. [CrossRef] [PubMed]

38. Bobrov, K.; Comtet, G.; Dujardin, G.; Hellner, L.; Bergonzo, P.; Mer, C. Surface Electronic States of the Partially Hydrogenated Diamond C(100)-(2x1):H Surface. Phys. Rev. B 2001, 63, 165421. [CrossRef]

39. Jaouen, M.; Tourillon, G.; Delafond, J.; Junqua, N.; Hug, G. A NEXAFS Characterization of Ion-Beam-Assisted Carbon-Sputtered Thin Films. Diam. Relat. Mater. 1995, 4, 200-206. [CrossRef]

40. Lenardi, C.; Baker, M.A.; Briois, V.; Coccia Lecis, G.; Piseri, P.; Gissler, W. Near-Edge X-Ray Absorption Fine Structure Study of Carbon Nitride Films. Surf. Coat. Technol. 2000, 125, 317-321. [CrossRef] 
41. Laikhtman, A.; Hoffman, A. Interaction of Thermally Activated and Molecular Oxygen with Hydrogenated Polycrystalline Diamond Surfaces Studied by Synchrotron Radiation Techniques. Surf. Sci. 2003, 522, L1-L8. [CrossRef]

42. Hoffman, A.; Comtet, G.; Hellner, L.; Dujardin, G.; Petravic, M. Surface Near-Edge x-Ray Adsorption Fine Structure of Hydrogenated Diamond Films and Di(100) Surfaces Studied by H+ and H- Ion Desorption. Appl. Phys. Lett. 1998, 73, 1152-1154. [CrossRef]

43. Parry, D.E. Atomic Calculation of Photoionization Cross-Sections and Asymmetry Parameters J.-J. YEH, Published by Gordon and Breach, Langhorne PA, 1993 ISBN 2-88124-585-4. Rapid Commun. Mass Spectrom. 1994, 8, 579. [CrossRef]

44. Stehlik, S.; Glatzel, T.; Pichot, V.; Pawlak, R.; Meyer, E.; Spitzer, D.; Rezek, B. Water Interaction with Hydrogenated and Oxidized Detonation Nanodiamonds-Microscopic and Spectroscopic Analyses. Diam. Relat. Mater. 2016, 63, 97-102. [CrossRef]

45. Ji, S.; Jiang, T.; Xu, K.; Li, S. FTIR Study of the Adsorption of Water on Ultradispersed Diamond Powder Surface. Appl. Surf. Sci. 1998, 133, 231-238. [CrossRef]

46. Shenderova, O.A.; Vlasov, I.I.; Turner, S.; Van Tendeloo, G.; Orlinskii, S.B.; Shiryaev, A.A.; Khomich, A.A.; Sulyanov, S.N.; Jelezko, F.; Wrachtrup, J. Nitrogen Control in Nanodiamond Produced by Detonation Shock-Wave-Assisted Synthesis. J. Phys. Chem. C 2011, 115, 14014-14024. [CrossRef]

47. Arnault, J. X-Ray Photoemission Spectroscopy Applied to Nanodiamonds: From Surface Chemistry to in Situ Reactivity. Diam. Relat. Mater. 2018. [CrossRef]

48. Chung, P.H.; Perevedentseva, E.; Cheng, C.L. The Particle Size-Dependent Photoluminescence of Nanodiamonds. Surf. Sci. 2007, 601, 3866-3870. [CrossRef]

49. Cheng, C.L.; Chen, C.F.; Shaio, W.C.; Tsai, D.S.; Chen, K.H. The CH Stretching Features on Diamonds of Different Origins. Diam. Relat. Mater. 2005, 14, 1455-1462. [CrossRef]

50. Ando, T.; Inoue, S.; Ishii, M.; Kamo, M.; Sato, Y.; Yamada, O.; Nakano, T. Fourier-Transform Infrared Photoacoustic Studies of Hydrogenated Diamond Surfaces. J. Chem. Soc. Faraday Trans. 1993, 89, 749-751. [CrossRef]

51. Williams, O.A.; Hees, J.; Dieker, C.; Jäger, W.; Kirste, L.; Nebel, C.E. Size-Dependent Reactivity of Diamond Nanoparticles. ACS Nano 2010, 4, 4824-4830. [CrossRef]

52. Petit, T.; Puskar, L. FTIR Spectroscopy of Nanodiamonds: Methods and Interpretation. Diam. Relat. Mater. 2018, 89, 52-66. [CrossRef]

53. Ozawa, M.; Inaguma, M.; Takahashi, M.; Kataoka, F.; Krüger, A.; Ōsawa, E. Preparation and Behavior of Brownish, Clear Nanodiamond Colloids. Adv. Mater. 2007, 19, 1201-1206. [CrossRef]

54. Shenderova, O.; Grichko, V.; Hens, S.; Walch, J. Detonation Nanodiamonds as UV Radiation Filter. Diam. Relat. Mater. 2007, 16, 2003-2008. [CrossRef]

55. Eidelman, E.D.; Siklitsky, V.I.; Sharonova, L.V.; Yagovkina, M.A.; Vul', A.Y.; Takahashi, M.; Inakuma, M.; Ozawa, M.; Ōsawa, E. A Stable Suspension of Single Ultrananocrystalline Diamond Particles. Diam. Relat. Mater. 2005, 14, 1765-1769. [CrossRef]

56. Reineck, P.; Francis, A.; Orth, A.; Lau, D.W.M.; Nixon-Luke, R.D.V.; Rastogi, I.D.; Razali, W.A.W.; Cordina, N.M.; Parker, L.M.; Sreenivasan, V.K.A.; et al. Brightness and Photostability of Emerging Red and Near-IR Fluorescent Nanomaterials for Bioimaging. Adv. Opt. Mater. 2016, 4, 1549-1557. [CrossRef]

57. Chang, S.L.Y.; Barnard, A.S.; Dwyer, C.; Boothroyd, C.B.; Hocking, R.K.; Ōsawa, E.; Nicholls, R.J. Counting Vacancies and Nitrogen-Vacancy Centers in Detonation Nanodiamond. Nanoscale 2016, 8, 10548-10552. [CrossRef] [PubMed]

58. Kaviani, M.; Deák, P.; Aradi, B.; Frauenheim, T.; Chou, J.P.; Gali, A. Proper Surface Termination for Luminescent Near-Surface NV Centers in Diamond. Nano Lett. 2014, 14, 4772-4777. [CrossRef] [PubMed]

59. Stacey, A.; Dontschuk, N.; Chou, J.P.; Broadway, D.A.; Schenk, A.K.; Sear, M.J.; Tetienne, J.P.; Hoffman, A.; Prawer, S.; Pakes, C.I.; et al. Evidence for Primal Sp2 Defects at the Diamond Surface: Candidates for Electron Trapping and Noise Sources. Adv. Mater. Interfaces 2019, 6, 1801449. [CrossRef]

60. Stacey, A.; Karle, T.J.; McGuinness, L.P.; Gibson, B.C.; Ganesan, K.; Tomljenovic-Hanic, S.; Greentree, A.D.; Hoffman, A.; Beausoleil, R.G.; Prawer, S. Depletion of Nitrogen-vacancy Color Centers in Diamond via Hydrogen Passivation. Appl. Phys. Lett. 2012, 100, 071902. [CrossRef] 
61. Hu, S.; Trinchi, A.; Atkin, P.; Cole, I. Tunable Photoluminescence Across the Entire Visible Spectrum from Carbon Dots Excited by White Light. Angew. Chem. Int. Ed. 2015, 54, 2970-2974. [CrossRef] [PubMed]

62. Baker, S.N.; Baker, G.A. Luminescent Carbon Nanodots: Emergent Nanolights. Angew. Chem. Int. Ed. 2010, 49, 6726-6744. [CrossRef] [PubMed]

(C) 2020 by the authors. Licensee MDPI, Basel, Switzerland. This article is an open access article distributed under the terms and conditions of the Creative Commons Attribution (CC BY) license (http:/ / creativecommons.org/licenses/by/4.0/). 